Research Article

\title{
Dental Anxiety Management by Full Spectrum CBD Formulations: Dual Dosing (AM/PM) Protocol in A Real-World Setting
}

\author{
Cooper DL', Stephan R and Maygar CW
}

PhytoDental Solutions LLC, Homosassa, Florida, 34446, USA

\begin{abstract}
Background: Dental Anxiety (DA) may produce a vicious cycle where dental problems are not adequately serviced. Chronic non-compliance with prescribed dental care and maintenance is associated ultimately with poor dental health. Current pharmacologic treatments such as benzodiazepines for DA are associated with poor efficacy and significant side effects. The anxiolytic effects of the most studied cannabinoid, cannabidiol (CBD) in the Dual Dosing (AM/PM) Full Spectrum Protocol are detailed here (DDFSP).
\end{abstract}

Materials and methods: Our recently developed PhytoDental Solutions Dental Anxiety Scale (PDSDAS) composed of eight self-reported psychic, somatic and sleep indicators was utilized for scoring a subject's DA level for three Time Groups (TG): the night before (T0); immediately after (T1); and 24 hours (T2) after a dental procedure or visit.

Results: In this series of subjects completing at least one post-dosing time point, the Dual Dosing AM/PM Full Spectrum CBD (DDFSCBD) Protocol significantly reduced both psychic ( $43 \%$ to $67 \%$ ) and somatic (51\%) dental anxiety. Additionally, $87 \%$ of study patients reported substantially improved sleep the night before their dental visit. Kruskai-Wallis one-way ANOVA analysis of the three Time Group data sets yielded significant 1-tail statistical differences ( $\mathrm{p}$-values $<0.05$ ) with p-values of 0.020 and 0.041 substantiating the role of the DDFSP to modify DA. Further, validation of the PDSDAS as a scoring measure developed for DA was extended by paired t-test comparisons to multiple smaller Paired Data sets across these Time Groups yielding 1-tail p-values of 0.010, 0.050 and 0.024 respectively.

Conclusion: Determination of significance by both ANOVA and paired t-tests of PDSDAS scoring strongly suggest the Dual-Dosing (AM/PM) Full Spectrum Protocol is an effective Dental Anxiety anxiolytic.

Keywords: Dental anxiety; Cannabinoid; Cannabidiol; Full spectrum; Anxiolytic; T-tests; P-value

\section{Abbreviations}

DA: Dental Anxiety; DDFSP: Dual Dosing (AM/PM) Full Spectrum Protocol; DDFSCBD: Dual Dosing Full Spectrum CBD; PDSDAS: PhytoDental Solutions Dental Anxiety Scale; CBD: Cannabidiol; ANOVA: Analysis of Variance; MDAS: Modified Dental Anxiety Scale; HAM-A: Hamilton Anxiety Rating Scale; ISI: Insomnia Severity Index; TG: Time Group Data; PD: Paired Data; CBDV: Cannabidivarian; THCV: Tetrahydrocannabivarian

\section{Introduction}

\section{Dental anxiety}

Anxiety is recognized as the number one mental illness in the world with associated financial and personal costs [1]. Anxiety associated with visiting a dentist for routine care or dental procedures is referred

Citation: Cooper DL, Stephan R, Maygar CW. Dental Anxiety Management by Full Spectrum CBD Formulations: Dual Dosing (AM/ PM) Protocol in A Real-World Setting Anesthesia and Patient Safety. Clin Med. 2021; 3(2): 1038.

Copyright: @ 2021 Cooper DL

Publisher Name: Medtext Publications LLC

Manuscript compiled: Nov $11^{\text {th }}, 2021$

*Corresponding author: David L Cooper, PhytoDental Solutions LLC, Homosassa, Florida, 34446, USA, Tel: 6086304622; E-mail: david@ phytodentalsolutions.com to as Dental Anxiety [2] (DA) and the fifth leading cause of anxiety overall [3]. Fully, up to $20 \%$ of dental patients suffer varying degrees of anxiety when faced with dental visits and procedures. Dental Anxiety is also a leading cause of dental health maintenance compliance failure [4] which multiple studies indicate correlates highly with subsequent dental disease [5]. Systemic disease associations in such patients is a further complication often referred to as having a multiplier effect [6].

\section{Pharmaceutical treatment}

Selective serotonin reuptake inhibitors, serotonin-norepinephrine reuptake inhibitors, monoamine oxidase inhibitors, tricyclics, partial 5-hydroxytryptamine 1A (5-HT1A) receptor agonists, and benzodiazepines are currently the pharmaceutical drug treatments available. Adverse effects and sub-optimal efficacy rates of only $40 \%$ to $60 \%$ in patients highlight the need for new therapeutic options [7].

\section{Cannabinoids in anxiety}

Utilization of cannabis and hemp extracts have been commonly utilized to treat anxiety in the far east for over 1000 years. However, only recently has modern scientific studies begun to critically evaluate this long believed property of the plant's molecular phytocannabinoid, terpene and flavonoid components. Characterization of the anxiolytic effects of the cannabinoids found in cannabis and hemp have focused mainly on CBD's activity in both rodents and humans. In pre-clinical rodent models of fear and anxiety-like behavior CBD has shown some evidence of anxiolytic, anti-stress, and anti-compulsive like effects [8]. In human trials CBD has shown in preliminary studies anxiolytic effects in individuals who suffer from social anxiety disorders $[9,10]$. 
Current scientific consensus on $\mathrm{CBD}$ and associated cannabinoids (CBC, CBG and others) as found in full spectrum formulations have considerable potential to treat multiple anxiety disorders [11]. The study described here has focused specifically on the potential anxiolytic effects in patients who suffer from mild to moderate to potentially severe dental anxiety in a real-world setting.

\section{Materials and Methods}

\section{Patient population}

A series of 12 patients selected by dental practice personnel as having exhibited mild to severe anxiety previously were consented and enrolled in this single arm study. This series of case studies conform to IRB designed studies for safety and efficacy consisting of and including appropriate sections on consent, trial protocol, medical history, medication log, and adverse event records. Eight of twelve patients completed the proposed study at least one time point past the baseline evaluation data. One subject upon medical review was considered to have among other psychological issues, dental phobia which was beyond the scope of this anxiolytic and therefore eliminated from data considerations as well.

\section{Formulation}

Patients were provided with two decarboxylated full spectrum hemp CBD oral formulations (i.e. Hempzorb81 $1^{\mathrm{mm}}$ ) containing CBD, Cannabidivarian (CBDV) and Tetrahydrocannabivarian (THCV). The PM dose formulated to assist with relaxation and sleep the night before included Valerian Root and Skullcap. The AM dose formulated to calm the patient the day of the procedure to be taken 15 minutes before arrival at the dentist's office contained a Vitamin B complex. Purzorb ${ }^{\circledR}$ is a proprietary micellular formulation rapidly absorbed with an onset of action within 15 minutes and with $85 \%$ bioavailability. Both formulations contain THC at less than $0.3 \%$ conforming to Federal regulations for $\mathrm{CBD}$ products.

\section{Dental anxiety determination}

PhytoDental Solutions Dental Anxiety Scale (PDSDAS): To measure the level of patient's Dental Anxiety the self-reporting PhytoDental Solutions Dental Anxiety Scale [12] (PDSDAS) was developed. The PDSDAS device was based on both the Modified Dental Anxiety Scale [13] (MDAS), the Hamilton Anxiety Rating Scale [14] (HAM-A) and the Insomnia Severity Index (ISI) [15]. The PDSDAS consists of eight self-reported items, tailored to measure the patient's psychic (anxiety before and while waiting) and somatic characteristics (sensory, cardiovascular, respiratory, gastrointestinal, autonomic) as well as sleeping when faced with a dental procedure in a brief self-reported manner. Scoring was achieved by assigning 0 points for not anxious to 4 points for extremely anxious to each of the 8 items depending upon the subject's self-evaluation at times T0, T1 and T2. Scoring was then tabulated into psychic and somatic characteristics. Each of the elements to evaluate anxiety have been validated independently previously [13-15] but not as a set of eight as put forth in our PDSDAS. A dental hygienist familiar with the patient's history reviewed the questionnaire and responses if clarification was required from the subject. Direct professional oversight thereby insured an increase in accuracy of the scoring.

Self-reporting empirics: Patients were asked to self-report their level of anxiety at three time points: a) the night before (T0) the dental visit b) in office immediately following (T1) dental procedure and c) 24 hours after (T2) the visit (Figure 1). The total scores from each time point divided by the number of patients was used as a measure to compare the overall population anxiety effect of the DualDosing (AM/PM) Protocol approach to "Breaking the Cycle of Fear: Managing patients with Dental Anxiety" [16,17] associated with a large percentage of patients in the course of their dental visit.

\section{Statistics}

AI-Therapy Statistics ${ }^{\text {BETA }}$, Statistics for Psychologists, ai-therapy. com was the statistical package utilized for analysis of this study's results. Statistics for Psychologists is a collection of web-based statistical analysis tools, visualizations and online calculators.

\section{Results}

\section{Subjects study enrollment and completion}

Eight of the twelve originally enrolled patients completed the study for at least one additional time point following Baseline PDSDAS scoring. Four patients did not fully participate for reasons of non-compliance or due to COVID-19 restrictions. One of the eight study complete subjects was removed for exhibiting behavior more consistent with dental phobia than dental anxiety. This study was designed to study dental anxiety from mild to severe.

\section{PDSDAS scoring}

In those completing the protocol, sufficiently to be scored by the PDSDAS, analysis of individual scores, comparing both the three time point data sets and internally developed same subject Data Sets our findings included the following (Table 1).

- Seven of eight patients reported overall decreased anxiety in symptoms, i.e. psychic or physical elements or both associated with the dental visit before and or afterwards.

- Psychic symptoms were reduced between $43 \%$ and $67 \%$.

- Somatic symptoms were reduced between $51 \%$.

- Markedly improved sleep was most notably reported in $87 \%$ subjects.

- 4 of 5 patients reported less total anxiety scores immediately following the dental procedure.

- 3 of 3 patients who were scored both immediately afterwards and 24 hours later reported experiencing less anxiety 24 hours after the procedure than immediately afterwards.

- One patient refused the AM dose because they felt the PM dose was so effective the patient needed nothing additionally for anxiety.

- 5 of 5 patients reported less total anxiety scores 24 hours following dental procedure.

- The one patient who did not report either better sleep or less anxiety reported an increased state of agitation and restlessness following the night time dose. This was the one and only potential side effect seen. This patient was originally identified by the ISI as having severe insomnia. This patient also reported feelings of psychic and somatic reflected maximum anxiety in all 5 of 5 categories rated by the PDSDAS the day of and following the dental visit. The patient was considered to be suffering from Dental Phobia, not Dental Anxiety and scores removed from the study.

\section{Statistical determination of significant difference}

Paired difference t-test: Classical determination of statistically 
DENTAL OFFICE

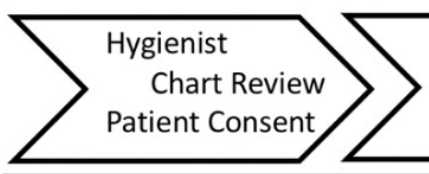

HOME

PM Dose

Full Spectrum CBD Before Bed

\section{Study Initiation}

PDSDAS Self Assessment

Baseline
DENTAL OFFICE

AM Dose

Full Spectrum CBD

15 Minutes before
HOME

PDSDAS Self Assessment PDSDAS Self Assessment Immediately After Visit 24 Hours Later

Group Time $=0$

Group Time $=1$

Group Time $=2$

Figure 1: Dual dosing (AM/PM) full spectrum CBD protocol and assessment in dental anxiety.

Table 1: PDSDAS raw subject scoring.

\begin{tabular}{|c|c|c|c|c|c|c|}
\hline \multirow{2}{*}{ PhytoDental Solutions Dental Anxiety Scale Scoring } & \multicolumn{2}{|c|}{ Time 0 - Baseline (Night before) } & \multicolumn{2}{|c|}{ Time - 1 (Immediately after) } & \multicolumn{2}{|c|}{ Time - 2 (24 hours later) } \\
\hline & Psychic & Somatic & Psychic & Somatic & Psychic & Somatic \\
\hline Subject Dental Anxiety Score & 26 & 50 & 6 & 18 & 8 & 18 \\
\hline Total Scale Score & 56 & 140 & 40 & 120 & 40 & 120 \\
\hline Subject Dental Anxiety Score/ Total Scale Scores & \multicolumn{2}{|c|}{$76 / 196$} & \multicolumn{2}{|c|}{$24 / 140$} & \multicolumn{2}{|c|}{$26 / 140$} \\
\hline Corrected value \% Change & 3.7 & 7.1 & $1.2(-67 \%)$ & $3.6(-51 \%)$ & $1.6(-43 \%)$ & $3.6(-51 \%)$ \\
\hline
\end{tabular}

In subjects completing at least one post-dosing time point, the Dual Dosing AM/PM Full Spectrum CBD Formulation approach significantly reduced psychic and somatic anxiety scores.

significant differences between two data sets most often relies on first performing a $\mathrm{t}$-test. The $\mathrm{t}$-test compares one set of measurements with a second sample from the same sample as in an experiment to test the safety or efficacy of a clinically useful formulation. Statistical significance between these sets in the before and after test indicates with some defined certainty a significant change has resulted. However, in many cases we may wish to determine if there are significant differences between more than two groups or sets of data. In our case we wished to understand how our test subjects perceived their DA treatment course.

Comparing more than two sets of data (ANOVA): Analysis of Variance (ANOVA) is a family of statistical tests useful to compare several sets of scored data. In this report our study defined three specific Groups of interest, i.e. T0, T1 and T2 (Table 2). The ANOVA approach allows us to compare each group to each other and any variance within a group as well as simultaneously. In our case we looked at whether a subject's view of how they perceived DA may change from preparing for the dental visit to 24 hours afterwards. Here we have a small data set to which the Shapiro-Wilk normality test is automatically applied by the AI-Therapy Statistics ${ }^{\mathrm{BETA}}$ package. Defining the three groups as independent allowed us to determine any significance that arose between DA assessments. A total of 119 PDSDAS scores were recorded across our three defined Time Group Data Sets.

Comparing paired data sets across time groups: As an alternative to testing groups against one another significant differences between

Table 2: Time groups data sets.

\begin{tabular}{|l|l|l|l|}
\hline & T0 & T1 & T2 \\
\hline Psychic (Score total / \# subject scores) & $26 / 14$ & $6 / 10$ & $8 / 10$ \\
\hline $\begin{array}{l}\text { Somatic/Sleep (Score total / \# subject } \\
\text { scores) }\end{array}$ & $50 / 35$ & $18 / 25$ & $18 / 25$ \\
\hline Total & $76 / 49$ & $24 / 35$ & $26 / 35$ \\
\hline 1-tail Kruskai-Wallis one-way ANOVA & \multicolumn{3}{|c|}{ 1-tail P-value 0.020 } \\
\hline 2-tail Kruskai-Wallis one-way ANOVA & \multicolumn{3}{|c|}{ 1-tail P-value 0.041 } \\
\hline $\begin{array}{l}\text { ANOVA Analysis Kruskai-Wallis one-way comparison of three Time Group } \\
\text { data sets yielded significant 1-tail statistical differences (p-values< 0.05). }\end{array}$
\end{tabular}

combinations of data sets within and across the groups may be valuable, though the process has room for more inherent associated error. We were interested in comparing specific sets of data over time due to the incompleteness of the data on all patients, i.e. lacking scores on all three time points. Utilizing the two data set comparison capability of the t-test did allow us to probe, in this preliminary study the perception of subjects between various time points individually of their feelings associated with DA. The Shapiro-Wilk test conducted on three of four Data Sets ((Table 3) Data Set\#1, Data Set\#2 and Data Set\#3) appeared by paired differences to be approximately normal. Our goal was to determine as a start whether our study design and execution would find p-values of 0.05 or less within-subject confidence limits of $95 \%$.

Data Set\#1: In this patient set the PDSDAS scoring data of the seven patients who had $\mathrm{T}=0$ night before time point and at least one follow-up time point $(\mathrm{T} 1$ or $\mathrm{T} 2)$ had a 1-tailed $\mathrm{p}$-value $=0.002$ based on significance level of $\mathrm{p}=0.05$. ((Table 3) Data Set\#1. PDSDAS Scoring T0 vs T1 or T2 Last).

Data Set\#2: Four of five patients reported less total anxiety scores immediately following the dental procedure PDSDAS scoring data of patients who had $\mathrm{T}=0$ night before time point and at immediate time point after (T1) had a 1-tailed p-value $=0.05$ based on significance level of $\mathrm{p}=0.05$. ((Figure 2) Data Set\#2PDSDAS Scoring T0 vs T1).

Data Set\#3: All five patients who self-reported anxiety scores 24 hours following dental procedure reported anxiety lessening when reflecting on this time ((Table 1), Data Set\#3). PDSDAS scoring data of the 5 patients who had $\mathrm{T}=0$ night before time point and $\mathrm{T} 2=24$ after time point's 1-tailed paired p-value of 0.012 to be normally distributed and therefore a significant difference based on significance level of 0.05. ((Table 3) Data Set\#3 PDSDAS Scoring T0 vs T2).

Data Set\#4: Three of three patients who were scored both immediately afterwards and 24 hours later reported experiencing less anxiety 24 hours after the procedure than immediately afterwards ((Table 1), Data Set\#4). PDSDAS scoring data of the 3 patients who had $\mathrm{T}=0$ night before time point and $\mathrm{T} 2=24$ after time point's 1 -tailed 
Table 3: Paired Data set comparisons for difference determination.

\begin{tabular}{|l|l|l|l|}
\hline \multicolumn{2}{|l|}{ Compared } & Difference Determination & P-value \\
\hline Data Set 1 & T0 with last (T1/T2) in subjects 1001,1002,1004.1005-8 & Last Time with Baseline & P-value 0.010 (T0 vs last time point T1/2) \\
\hline Data Set 2 & T0 with T1 in subjects 1001, 1002,1004,1005,1007 & Immediately After & P-value 0.050 (T0 v T1) \\
\hline Data Set 3 & T0 with T2 in subjects 1002, 1004,1005,1007 & 24 Hours After & P-value 0.024 (T0/T2) \\
\hline Data Set 4 & T1 with T2 in subjects 1002, 1004, 1005 & Immediate to 24 Hours & $\begin{array}{l}\text { P-value 0.019 (T2/T1) distribution, and numbers not } \\
\text { sufficient. }\end{array}$ \\
\hline
\end{tabular}

Significant statistical differences ( $\mathrm{p}$-value $<0.05$ ) are found in comparing each subject's paired differences with themselves and various data sets constructed to test significance of Dual Dosing Protocol on modifying DA.

paired p-value of 0.019 to be potentially not normally distributed and therefore a non-significant difference based on significance level of 0.05. ((Table 3) Data Set\#4 PDSDAS Scoring T1 vs T2).

\section{Discussion}

In psychology research to test whether or not two data sets differ on a particular variable is an invaluable measure of a unique approach where etiology of the activity may be complex. For example, it may be very useful, as here to determine if there is a statistically significant difference between anxiety levels before and after a proposed treatment strategy. There are a number of tests available for comparing two data sets, depending on the nature of the data and the experimental design. In our case we provide in this paper a smaller number of cases than planned due to the continued COVID-19 pandemic in the U.S. and Florida. However, in breaking the study at this early half way point, if the distribution of data points is sufficiently similar with approximate equal variance between our groups and identified Data Sets are identified, especially early on, such data is worth considerable evaluation. An advantage in this preliminary study was the utility of having the ability to confer statistical significance by both utilization of same subjects at multiple time points allowing for the statistical approach to derive differences between paired scores as well as using raw scores by utilizing our groups non-identical populations.

The expectation of our Dual-Dousing Dental Anxiety management approach was for a result in one direction, i.e. less anxiety. Although we did expect treatment to reduce anxiety, due to the cannabinoid family of molecules role in modulating homeostatic regulation a possibility of both tails being important remain. This proved out to be a correct assumption given the significant $p$-values found for the 1-tail paired t-tests on all but one of the four Data Sets. Similarly, ANOVA comparison of all three Time Group data sets simultaneously where we set out to evaluate both 2-tail and 1-tail scenarios twice achieved significant 1-tailed p-values of 0.020 and 0.041 .

A potential noteworthy and unexpected result to follow is the viewing of the anxiety level experienced as lessening over time after the procedure by all 5 patients in Data Set\#3 who self-reported at 24 hours and overlapping Data Set\#4 (3 of 3 completed all three-time point scoring) similarly reporting viewing earlier anxiety as lessened over time. Patient reports such as these noting a continued decrease in anxiety post visit may indicate less anxiety may accompany future dental visits as a result of the effective anxiolytic AM/PM dual dosing regimen just experienced. Evaluating these patients before their next scheduled dental visit may confirm in part this assumption.

Dental fear may produce a vicious cycle where dental problems are not adequately serviced as the patient no longer can face the symptom-driven treatment when faced with the threatening stimuli perceived with in-office dental care and procedures. The Dual Dosing (AM/PM) Protocol is PhytoDental Solutions approach to Dental Anxiety Management. Data from this preliminary study illustrates the ability of the full spectrum hemp formulations utilized here to have the potential to "break" the Cycle of Dental Fear [13].

\section{Conclusion}

In this preliminary study's early look, subjects completing at least one-post dosing time point of the Dual Dosing AM/PM Full Spectrum CBD formulation protocol reported reduced psychic (43\% to 67\%) and somatic $(51 \%)$ dental anxiety characterizations. Additionally, $87 \%$ (7 of 8 ) subjects reported substantially reduced night Dental Anxiety before their following day visit/procedure.

PDSDAS scoring revealed statistically significant differences between the three Time Groups ((Table2) T0,T1 and T2) with p-values of 0.020 and 0.041 and three of four developed Data Sets ((Table 3) Data Set\#1, Data Set\#2 and Data Set\#3) with 1-tailed paired t-tests reported p- values of 0.002 and 0.05 and 0.024 strongly suggesting the two-dose formulation strategy in concert may be an effective anxiolytic for dental anxiety. Based on these positive initial results an appropriately sized two-arm, placebo controlled, a blinded Pilot Study for Institutional Review Board approval is in preparation. The follow up study will be utilized to study dental anxiety not only across a real-world dental practice's response to the Dual-Dosing (AM/ PM) Protocol but also to further validate the PDSDAS as a useful diagnostic and monitoring tool going forward.

\section{Author's Note}

During the publishing process of this manuscript the dental practice is experiencing patients and patient's families who were part of this study now asking for the availability of the Full Spectrum AM/PM Formulations for their next upcoming scheduled visit.

\section{Acknowledgement}

The authors, especially the mad scientist Dr. Cooper would like to thank the dental hygienists and office staff of Dr. Maygar's Homosassa and Lecanto Offices who enthusiastically integrated this study into their busy work day.

\section{References}

1. Scherma M, Masia P, Deidda M, Fratta W, Tanda G, Fadda P. New perspectives on the use of cannabis in the treatment of psychiatric disorders. Medicines (Basel). 2018;5(4):107.

2. Appukuttan DP. Strategies to manage patients with dental anxiety and dental phobia: literature review. Clin Cosmet Investig Dent. 2016;8:35-50.

3. Agras S, Sylvester D, Oliveau D. The epidemiology of common fears and phobia. Compr Psychiatry. 1969;10(2):151-6.

4. Gatchell RJ, Ingersoll BD, Bowman L, Robertson MC, Walker C. The prevalence of dental fear and avoidance: a recent survey study. J Am Dent Assoc. 1983;107(4):60910 .

5. Locker D, Liddell A. Clinical correlates of dental anxiety among older adults. Community Dent Oral Epidemiol.1992;20(6):372-5.

6. Chi AC, Neville BW, Krayer JW, Gonsalves WC. Oral manifestations. Am Fam Physician. 2010;82(11):1381-8.

7. Craske MG, Stein MB, Eley TC, Milad MR, Holmes A, Rapee RM, et al. Anxiety 
disorders. Nat Rev Dis Primers. 2017;3:17024.

8. Tambaro S, Bortolato M. Cannabinoid-related agents in the treatment of anxiety disorders: current knowledge and future perspectives. Recent Pat CNS Drug Discov. 2012;7(1):25-40

9. Zuardi AW, Cosme RA, Graeff FG, Guimaraes FS. Effects of ipsapirone and cannabidiol on human experimental anxiety. J Psychopharmacol. 1993;7:82-8.

10. Bergamaschi MM, Queiroz RH, Chagas MH, Kapczinski F, Quevedo J, Roesler R, et al Cannabidiol reduces the anxiety induced by simulated public speaking in treatmentnaive social phobia patients. Neuropsychopharmacology. 2011;36(6):1219-26.

11. Wright M, Di Ciano P, Brands B. Use of Cannabidiol for the Treatment of Anxiety: A Short Synthesis of Pre-Clinical and Clinical Evidence. Cannabis and Cannabinoid Research. 2020;5(3):191-6.
12. Note: The PhytoDental Anxiety Score (PDSAS) has not been independently validated. Manuscript in preparation.

13. Humphris GM, Morrison T, Lindsay SJ. The Modified Dental Anxiety Scale: Validation and United Kingdom norms. Community Dent Health. 1995;12(3):143-50.

14. Hamilton M. The assessment of anxiety states by rating. Br J Med Psychol. 1959;32(1):50-5

15. Morin Charles M, Belleville G, Belanger L, Ivers H. The Insomnia Severity Index: psychometric indicators to detect insomnia casesand evaluate treatment response. Sleep. 2011; 34(5):601-8

16. Armfield JM, Stewart JF, Spencer AJ. The vicious cycle of dental fear: exploring the interplay between oral health, service utilization and dental fear. BMC Oral Health. 2007;7:1.

17. Cooper, DL, Maygar CW. "Breaking the Cycle of Fear: Managing Patients with Dental Anxiety." Manuscript in preparation. 